\title{
Prevalence of malaria plasmodium in Abeokuta, Nigeria
}

\author{
Okonko, I. O. ${ }^{1 \star}$, Soleye, F. A. ${ }^{2}$, Amusan, T. A. ${ }^{3}$, Ogun, A. A. ${ }^{4}$, Udeze, A. O. ${ }^{5}$, Nkang, A. O. ${ }^{1}$, Ejembi, J. ${ }^{6}$ and Faleye, \\ T. O. C. \\ ${ }^{1}$ Department of Virology, Faculty of Basic Medical Sciences, University of Ibadan College of Medicine, University College \\ Hospital (UCH), Ibadan, University of Ibadan, Ibadan, Nigeria. WHO Regional Reference Polio Laboratory, WHO \\ Collaborative Centre for Arbovirus Reference and Research, WHO National Reference Centre for Influenza, National \\ HIV Reference Laboratory. \\ ${ }^{2}$ Medical Laboratory Unit, Department of Health Services, University of Agriculture, Abeokuta, P.M.B. 2240, Abeokuta, \\ Ogun State, Nigeria. \\ ${ }^{3}$ Department of Veterinary Biochemistry, College of Veterinary Medicine, University of Agriculture, Abeokuta, Ogun State, \\ Nigeria. \\ ${ }^{4}$ Department of Epidemiology, Medical Statistics and Environmental Health, Faculty of Public Health, College of \\ Medicine, University of Ibadan, Ibadan, Nigeria. \\ ${ }^{5}$ Department of Microbiology, Faculty of Sciences, University of Ilorin, Ilorin, Nigeria. \\ ${ }^{6}$ Department of Clinical Microbiology, Ahmadu Bello University Teaching Hospital (ABUTH), Zaria, Kaduna State, Nigeria. \\ E-mail:mac2finney@yahoo.com
}

Received 16 February 2009; received in revised form 3 July 2009; accepted 6 July 2009

\begin{abstract}
This study reports the prevalence of malaria caused by plasmodium between genders in Abeokuta, the capital city of Ogun State located in the forest zone of southwestern Nigeria between January 2002 and December 2004. Blood film examination for malaria parasites in 708 patients; 366 males and 342 females. Microscopic examination of thick films techniques was employed for this study. Of the 708 (100\%) patients examined, 577 (81.5\%) were Plasmodium-positive. A high malaria parasite prevalence rate of $81.5 \%$ was noted in this study. Female subjects were more infected (42.4\%) than males (41.9\%) however, there was no significant difference in the sex of the subjects studied ( $p=0.05)$. A high malaria parasite prevalence rate of $86.9 \%$ was noted in samples collected in year 2003 than in other years studied. There was significant difference in the years under study $(p=0.05)$. This study shows that a good percentage of people were infested by malaria Plasmodium. This could be attributed to lack of adequate accommodation and poor sanitary conditions in the area under study. Although several efforts have been made to effectively control the high incidence of malaria in Nigeria, these have been largely unsuccessful due to a number of reasons such as irrigated urban agriculture which can be the malaria vector's breeding ground in the city, stagnant gutters and swamps in our environment where mosquitoes breed in millions, and lack of political will and commitment of the government in its disease management program, low awareness of the magnitude of malaria problem, poor health practices by individuals and communities and resistance to drugs. Therefore, future interventions in Nigeria should be directed toward controlling malaria in the context of a moderate transmission setting; thus, large-scale distribution of insecticide-treated nets or widespread use of indoor residual spraying may be less cost-effective than enhanced surveillance with effective case management or focused larval control.
\end{abstract}

Keywords: mosquito eradication, malaria, malaria interventions, plasmodium

\section{INTRODUCTION}

Malaria is the world's most deadly parasitic disease and is caused by infection with single-celled parasites of the genus Plasmodium belonging to the apicomplexan phylum Anopheles mosquitoes transmit these parasites from one person to another in their bites (Microsoft Encarta, 2009). Malaria is characterized by periodic bouts of severe chills and high fever. Serious cases of malaria can result in death if left untreated. More than a million people die of the disease each year, most of them in Africa, according to the World Health Organization (WHO) (Microsoft Encarta, 2009). It is one of the most prevalent and deadly widespread of all parasitic diseases in the world. Over 500 million people suffer clinical malaria episodes annually caused by Plasmodium falciparum infection alone resulting in a conservative estimate of 1 million deaths (Guinovart et al., 2006; Vaughan et al., 2008).

Malaria was once widespread in North America and other temperate regions. Today, the disease occurs mostly in tropical and subtropical regions, particularly in sub-Saharan Africa and Southeast Asia. The disease is also found in Central and South America, Oceania, and on some Caribbean islands. Public health officials had hoped to wipe out malaria during the $20^{\text {th }}$ century. However, malaria parasites have developed defenses against many antimalarial drugs. This response, known as drug resistance, makes the drugs less effective. In addition, the 
Anopheles mosquitoes that transmit the disease have become resistant to many insecticides (Microsoft Encarta, 2009).

Indeed, malaria is still remains one of the most significant public health problems in Nigeria and perhaps the commonest cause of ill health in Africa. Almost all the entire population of Africa is at risk of this parasitic disease that has continued to claim at least one million deaths. It is mosquito-borne and one of the killer diseases of the world, currently accounting for about 300 to 500 million clinical cases annually and over 1.2 to 2.7 million deaths worldwide each year (WHO, 1992). In Nigeria, statistics show that malaria accounts for $25 \%$ of under-five mortality, $30 \%$ of childhood mortality and $11 \%$ of maternal mortality. All Nigerians are at risk of malaria and the problem is compounded by the increasing resistance of malaria to hitherto cost-effective antimalaria drugs.

Previous studies have shown that Anopheles mosquito breeding decreases with increasing proximity to the center of urban areas (Robert et al., 2003). Although the complex factors that contribute to malaria risk are not fully understood, availability of vector breeding sites is clearly essential (Robert et al., 2003). Urban agriculture, promoted as a means of increasing food security, improving nutrition, and alleviating poverty, can, especially when irrigated, create breeding habitats that could increase malaria transmission in cities. This potential risk was indicated by other authors (Afrane et al., 2004).

However, malaria remains a global health problem, and public health efforts today focus on controlling it. In addition, a worldwide effort is under way to develop a vaccine that protects people against the disease. In the meantime, research by the WHO has found that sleeping under bed nets treated with insecticide can greatly reduce deaths from malaria, especially among children (Microsoft Encarta, 2009). Over the years, a lot of efforts have gone into controlling malaria in Nigeria and other African countries, the most affected by the disease, but the problem has not shown any sign of abating. The reasons for the limited success in efforts to eradicate malaria-a disease of poverty in Nigeria include lack of political will and commitment, low awareness of the magnitude of malaria problem, poor health practices by individuals and communities and resistance to drugs (Yusuf, 2007). This present study reports the prevalence of malaria caused by plasmodium between genders in Abeokuta, the capital city of Ogun State located in the forest zone of southwestern Nigeria between January 2002 and December 2004.

\section{MATERIALS}

Microscopy is the main tool for laboratory diagnosis of malaria (WHO, 1992). The thick blood film and the thin blood film methods are employed. Field's stain A (A polychrome methylene blue, disodium hydrogen phosphate and potassium hydrogen phosphate) and Field's stain B (Eosin, disodium hydrogen phosphate and potassium hydrogen phosphate). Thin film is carried out following the examination of thick film to identify the particular species of the Plasmodium responsible for the infection; it is stained with commercially prepared Leishman stain.

The materials employed in the study included a Leitz light microscope, EDTA (ethylene diamine tetra acetic acid) bottles, methylated spirit (methanol), cotton wool, tourniquet, syringes $(5 \mathrm{~mL}$ ) and needles $(21 \mathrm{G})$ (Epidi et al., 2008).

\section{Study area}

This study was carried out at the Department of Health Services, University of Agriculture, Abeokuta. Abeokuta is the capital city of Ogun State located in the forest zone of southwestern Nigeria between January 2002 and December 2004.

\section{Study population}

After informed consent was obtained, a total of 708 subjects; (366 males and 342 females) blood samples were collected from subjects attending the Department of Health Services, University of Agriculture, Abeokuta, South-Western, Nigeria. Thick blood films were prepared for each person.

\section{METHODS}

\section{Sample collection}

The method of sample collection employed was venepuncture technique (Carmel et al., 1993; Ibhanesebhor et al., 1996; Okocha et al., 2005; Epidi et al., 2008). Soft tubing tourniquet was fastened to the upper arm of the patient to enable the index finger feel a suitable vein. The puncture site was then cleansed with methylated spirit (methanol) and venepuncture made with the aid of a $21 \mathrm{G}$ needle attached to a $5 \mathrm{~mL}$ syringe. When sufficient blood had been collected, the tourniquet was released and the needle removed immediately while the blood was transferred into an EDTA bottle (Epidi et al., 2008).

\section{Laboratory Analysis}

The collected blood samples were analyzed within 1 to $2 \mathrm{~h}$ of collection. Thick and thin blood films were prepared according to the technique outlined by Cheesebrough (2004) and described by Epidi et al. (2008). A drop of each blood sample was placed in the center of a greasefree clean glass slide. Thereafter, the reverse side of the slide was cleaned with cotton wool and kept for air-drying and staining with field's stain. The slide was held with the dried thick film side facing downward and dipped in field's stain $A$ (eosin) for $5 \mathrm{~s}$. It was washed off gently in clean water and then dipped in field's stain B (methyl azure) for $5 \mathrm{~s}$ and washed again in clean water. The back of the slide was cleaned with cotton wool and kept in the draining rack to air-dry for eventual examination under the microscope, using oil immersion at $100 \mathrm{X}$ magnification to observe for Plasmodium parasites. Presence of ring forms 
of Plasmodium and Trophozoites of Plasmodium indicate positive results. A blood smear was considered negative if no parasite seen after 10 min of search or examination under 100 high power fields of microscope.

\section{Identification}

Positive specimens were identified on the basis of microscopy. Using standard methods (CDC, 2007), a trained laboratory technician at UNAAB Health Services Department interpreted the malaria blood slides. Prevalence of Plasmodium was calculated as the proportion of sampled persons with a positive result divided by the number of persons who provided blood samples. All point estimates were weighted, with empirically estimated standard errors used to account for prevalence.

\section{Data analysis}

The data generated from this study were presented using descriptive statistics. With SPSS version 15.0 for Windows (SPSS, 2006), was used to evaluate whether or not there were associations between the malaria Plasmodium prevalence and the subjects' variables. SPSS computer software was used for data analysis. Confidence level was set at $p=0.05$

\section{RESULTS}

A total of 708 persons from different households in different communities and locations were enrolled in the study. Microscopy at UNAAB Health Services Department identified 577 (81.5\%) malaria infections among the 708 $(100.0 \%)$ persons who had provided a blood sample. Presence of ring forms of Plasmodium and Trophozoites of Plasmodium indicate positive results. The malaria plasmodium of these 708 blood samples collected from apparently healthy persons in this study with Plasmodium positive and -negative slides are shown in Table 1. Table 1 shows the plasmodium of the apparently healthy persons in Abeokuta, Nigeria from 2002 to 2004. This study shows $267(80.7 \%)$ cases of malaria Plasmodium among the subjects in 2002; $133(86.9 \%)$ cases in 2003 and $177(79.0 \%)$ in 2004 (Table 1).

Table 2 shows the frequency and distribution of malaria Plasmodium between genders of apparently healthy subjects from 2002 to 2004 . The distribution of the Plasmodium species found in the 577 (81.5\%) positive subjects is shown in Table 2; of which 297 (41.9\%) of the male subjects tested positive for Plasmodium and 300 $(42.4 \%)$ of the female subjects had Plasmodium (Table 2 ).

Table 3 shows the frequency and distribution of malaria Plasmodium between genders of apparently healthy subjects by year (2002). The distribution of the malaria Plasmodium found in $331(100.0 \%)$ subjects is shown in Table 3; of which $267(80.7 \%)$ of them were positive for Plasmodium, $134(85.9 \%)$ of the positive subjects were males and $133(76.0 \%)$ were females as shown in Table 3.
Table 4 shows the frequency and distribution of malaria Plasmodium between genders of apparently healthy subjects by year (2003). The distribution of the Plasmodium species found in $153(100.0 \%)$ subjects for the year 2003 is shown in Table 5; of which 133 (86.9\%) of them were positive for Plasmodium; $55(83.3 \%)$ of the positive subjects were males and 78 (89.7\%) were females as shown in Table 4.

Table 1: Malaria Plasmodium among apparently healthy subjects from 2002 to 2004

\begin{tabular}{cccc}
\hline Year & No. tested (\%) & $\begin{array}{c}\text { No. positive } \\
(\%)\end{array}$ & $\begin{array}{c}\text { No. negative } \\
(\%)\end{array}$ \\
\hline 2002 & $331(46.8)$ & $267(80.7)$ & $64(19.3)$ \\
2003 & $153(21.6)$ & $133(86.9)$ & $20(13.1)$ \\
2004 & $224(31.6)$ & $177(79.0)$ & $47(20.9)$ \\
\hline Total & $708(100.0)$ & $577(81.5)$ & $131(18.5)$ \\
\hline
\end{tabular}

Table 2: Distribution of malaria Plasmodium between genders of apparently healthy subjects from 2002 to 2004

\begin{tabular}{ccccc}
\hline \multirow{2}{*}{ Year } & \multirow{2}{*}{$\begin{array}{c}\text { No. } \\
\text { tested }\end{array}$} & \multicolumn{2}{c}{ No. positive (\%) } & No. males \\
\cline { 3 - 4 } & & \multicolumn{1}{c}{$\begin{array}{c}\text { No. females } \\
(\%)\end{array}$} & \\
\hline 2002 & 331 & $134(40.5)$ & $133(40.2)$ & $267(80.7)$ \\
2003 & 153 & $55(35.9)$ & $78(50.9)$ & $133(86.8)$ \\
2004 & 224 & $97(43.3)$ & $80(35.7)$ & $177(79.0)$ \\
\hline Total & 708 & $297(41.9)$ & $300(42.4)$ & $577(81.5)$ \\
\hline
\end{tabular}

Table 3: Frequency and distribution of malaria Plasmodium between genders of apparently healthy subjects by year 2002

\begin{tabular}{cccc}
\hline Sex & $\begin{array}{c}\text { No. tested } \\
(\%)\end{array}$ & $\begin{array}{c}\text { No. positive } \\
(\%)\end{array}$ & $\begin{array}{c}\text { No. negative } \\
(\%)\end{array}$ \\
\hline Males & $156(47.1)$ & $134(85.9)$ & $22(14.1)$ \\
Females & $175(58.9)$ & $133(76.0)$ & $42(24.0)$ \\
\hline Total & $331(100.0)$ & $267(80.7)$ & $64(19.3)$ \\
\hline
\end{tabular}

Table 4: Frequency and distribution of malaria Plasmodium between genders of apparently healthy subjects by year 2003

\begin{tabular}{cccc}
\hline Sex & $\begin{array}{c}\text { No. Tested } \\
(\%)\end{array}$ & $\begin{array}{c}\text { No. Positive } \\
(\%)\end{array}$ & $\begin{array}{c}\text { No. Negative } \\
(\%)\end{array}$ \\
\hline Males & $66(43.1)$ & $55(83.3)$ & $11(16.7)$ \\
Females & $87(56.9)$ & $78(89.7)$ & $9(10.3)$ \\
\hline Total & $153(100.0)$ & $133(86.9)$ & $20(13.1)$ \\
\hline
\end{tabular}


Table 5: Frequency and distribution of malaria Plasmodium between genders of apparently healthy subjects by year 2004

\begin{tabular}{cccc}
\hline Sex & $\begin{array}{c}\text { No. tested } \\
(\%)\end{array}$ & $\begin{array}{c}\text { No. positive } \\
(\%)\end{array}$ & $\begin{array}{c}\text { No. negative } \\
(\%)\end{array}$ \\
\hline Males & $120(53.6)$ & $97(80.8)$ & $23(19.2)$ \\
Females & $104(46.4)$ & $80(76.9)$ & $24(23.1)$ \\
\hline Total & $224(100.0)$ & $177(79.0)$ & $47(21.0)$ \\
\hline
\end{tabular}

Table 5 shows the frequency and distribution of malaria Plasmodium between genders of apparently healthy subjects by year (2004). The distribution of the Plasmodium species found in 224 (100.0\%) subjects for the year 2004 is shown in Table 5; of which $177(79.0 \%)$ of them were positive for Plasmodium; 97 (80.8\%) of the positive subjects were males and 80 (76.9\%) were females as shown in Table 5.

\section{DISCUSSION}

Malaria occurs almost exclusively in the tropics and subtropics (WHO, 2006) and approximately, $40 \%$ of the world's population, mostly those living in the world's poorest countries, are at risk of malaria (Atif et al., 2009). Every year, more than 500 million people become severely ill with malaria-most cases and deaths occur within sub-Saharan Africa (WHO, 2007).

In this study, the presence of ring forms of Plasmodium and Trophozoites of Plasmodium indicate positive results. Results from the survey showed the overall prevalence of Plasmodium infection from 2002 to 2004 to be $85.1 \%$ in this area of Abeokuta, Southwestern, Nigeria. This differ from the overall prevalence of $59.9 \%$ reported in a study by Ojo and Mafiana (2005) among children $<15$ years in Abeokuta, Southwestern Nigeria and $51.5 \%$ reported by Epidi et al. (2008) among blood donors in Abakaliki, Southeastern Nigeria. Our results are substantially higher than previous estimates from passive surveillance of suspected malaria case-patients (Beatty et al., 2007). Atif et al. (2009) reported an incidence rate of $10.5 \%$ malaria infection in a similar study among 1000 patients in Hyderabad, Sind, Pakistan.

Prevalence rate of $81.5 \%$ in this study represents a substantial level of illness, especially when one considers that the severity of the disease is likely high given the low level of acquired immunity among this population (Klinkenberg et al., 2005). This high prevalence underscores the fact that, malaria is still a heavy burden on the continent, despite all that has been done.

Malaria can affect all the age humans groups and both male and female sexes. Studies have also shown seasonal variations in the rate of infections and differences in the types of malarial parasite depending upon the geographical conditions (Ghulam et al., 2004). This study also showed that malaria parasite (Plasmodium) was higher in females $(42.4 \%)$ than in male $(41.9 \%)$ subjects. This is in agreement with the findings of lbekwe
(2004) and Ibekwe et al. (2009) in similar studies in Southeastern, Nigeria but differs from Atif et al. (2009) who reported infection rate to be higher among young adult males in Pakistan.

This study shows that a good percentage of the apparently healthy persons were infested by malaria with Plasmodium. Majority of the subjects belonged to urban areas of Abeokuta and they were educated with higher socio-economic class. This is also contrary to Atif et al. (2009) who reported malaria infections to be prevalent among majority of uneducated patients with lower socioeconomic class belonging to the rural areas of Hyderabad, in Pakistan.

The prevalence of Plasmodium is attributed to its ability to resist attack of most drugs that are commonly in use in the study area. The stagnant drainage systems in the University, Abeokuta Metropolis and its environs created favourable environmental conditions for the breeding of mosquitoes that act as vectors of malaria parasites and so this enhances the proliferation of the Plasmodium species. The prevalence of the plasmodium among these subjects could also be attributed to the effect of climatic features on vector breeding and transmission as wet season usually promote mosquito breeding.

The high prevalence of malaria Plasmodium obtained in this study is worrisome because high-density urban African populations are not often considered particularly vulnerable to malaria infection. In other West African urban areas, malaria prevalence rates from $2 \%$ to $16 \%$ have been reported with large variation between communities (Sabatinelli et al., 1986). According to Klinkenberg et al. (2005), the observed low level of sensitivity of microscopy for identifying Plasmodium infections is similar to findings observed elsewhere (Kasehagen et al., 2006). This could be attributable to 2 factors: 1) many of the infections likely occurred at low parasite densities, and 2) the laboratory technician was responsible for reading a large number of slides with low parasite prevalence over a relatively short period.

Malaria transmission was highly localized; all 577 $(81.5 \%)$ infections were in persons from different areas of Abeokuta, which suggest that transmission is potentially based on a set of discrete ecologic determinants. Such clustering is consistent with the observed tendency for Anopheles mosquitoes to over disperse (Klinkenberg et al., 2005). Recently, several authors focused attention on urban malaria (Robert et al., 2003) and stressed the need to investigate risk factors for urban malaria. Although levels of transmission in urban areas may be lower than in contiguous rural areas, high population densities and possible lower immunity (Trape and Zoulani, 1987) may result in more disease impact in urban settings. Furthermore, although not the sole cause, irrigated urban agriculture which is practiced at the university may further increase the risk for malaria by providing suitable breeding sites.

The human behavioural pattern is a major epidemiological factor that impacts on disease transmission and progression in Africa and there is growing evidence that with appropriate awareness, 
education, attitude, attention to and chemotherapy of, the key symptoms of malaria, the incidence of severe malaria can be drastically reduced especially in the rural and urban areas where most of the estimated 2 to 3 million deaths per year from malaria occur (Miller et al., 1994; WHO, 2000). Prompt and accurate diagnosis of malaria is the key to effective disease management and therefore it is one of the main interventions of the global malaria control strategy (WHO, 1993). Identification of the species of malarial parasite is very important for its effective and curative treatment as resistance to chloroquine and other anti-malarial drugs has been reported previously (Rahim and Younas, 2003; Muhammad et al., 2004; Atif et al., 2009). Malaria in pregnancy is significantly associated with higher mortality and morbidity including cerebral malaria, maternal malaria, intrauterine growth retardation, abortions, still birth and premature labour (Kochar et al., 1998; Sullivan et al., 1999; Atif et al., 2009).

Although several efforts have been made to effectively control the high incidence of malaria in Nigeria, these have been largely unsuccessful due to a number of reasons such as lack of political will and commitment, low awareness of the magnitude of malaria problem, poor health practices by individuals and communities and resistance to drugs (Sambo, 2007). Consequently, as long as there are stagnant gutters and swamps in our environment where mosquitoes breed in millions, there shall be no respite to the malaria scourge and its attendant effect on the health and socio-economic life of Nigerians and by extension Africans (Yusuf, 2007). Reducing poverty and improving sanitation and access to health care in malaria endemic regions would go a long way to reduce the malaria burden in Africa (Atif et al., 2009). For those living in malaria endemic countries, limited resources frequently makes malaria prevention very difficult to implement. Vector control (reducing the breeding grounds by spraying or destruction of habitat) has only had very limited success. More successful strategies could include (WHO, 2005): Use of insecticidetreated bed nets (ITNs), indoor residual spraying, targeted chemoprophylaxis for those most at risk for pregnant women and travelers (Atif et al., 2009)

In light of the current progress of malaria control efforts in Nigeria, where most states are not malaria free and the total number of cases has been steadily increasing, Nigeria is not yet on its way to achieving those original eradication goals. A key aspect of future research in Nigeria should therefore focus on understanding treatment-seeking behavior, barriers to accessing health services among febrile persons, and quantifying patterns of malaria transmission (Klinkenberg et al., 2005). Future malaria intervention and preventive measures for the future hopes in the development of fatal malaria should include reducing poverty and improving access to health care in malaria endemic regions in Africa (Suh et al., 2004). According to Suh et al. (2004) and Atif et al. (2009), malaria prevention for effective result should include measures taken both against mosquitoes' vectors and against the malarial parasite. Such interventions include vector control programs managed by government health authorities, personal protection measures to avoid mosquito bites and the use of chemoprophylaxis. However, due to the development of drug resistant parasites, drug side-effects and contraindications, the control of vector mosquitoes and avoidance of their bites have become increasingly important (Suh et al., 2004; Atif et al., 2009). Furthermore, the development of new antimalarial drugs for prophylaxis and treatment as well as vaccines against malaria is also one important area under grand challenge exploration and the Malaria Genome project researches which involve molecular manipulation of the mosquito genome to produce transgenic mosquitoes that cannot infect humans will hopefully to provide new targets for both drugs and vaccines against malaria infection.

Nonetheless, future malaria interventions in Nigeria should also be directed toward controlling malaria in the context of a moderate transmission setting; thus, largescale distribution of insecticide-treated nets or widespread use of indoor residual spraying may be less cost-effective than enhanced surveillance with effective case management or focused larval control.

\section{REFERENCES}

Afrane, Y. A., Klinkenberg, E., Drechsel, P., OwusuDaaku, K., Garms, R., and Kruppa, T. (2004). Does irrigated urban agriculture influence the transmission of malaria in the city of Kumasi, Ghana? Acta Tropica 89, 125-134.

Atif, S. H., Farzana, M., Naila, S., and Abdul, F. D. (2009). Incidence and pattern of malarial infection at a tertiary care Hospital of Hyderabad. World Journal of Medical Sciences 4, 9-12.

Beatty, M. E., Hunsperger, E., Long, E., Schurch, J., Jain, S., and Colindres, R. (2007). Mosquitoborne infections after Hurricane Jeanne, Haiti, 2004. Emerging Infectious Diseases 13, 308-310.

Carmel, B., Kenmogne, D., Copin, N., and Mbitsi, A. (1993). Plasmodium prevalence and parasite burden in blood donors of Brazzaville, Congo. Annal of Society for Belgium Medicine Tropical 73, 179-187.

Centers for Disease Control and Prevention (2007). Laboratory identification of parasites of public health concern. $27^{\text {th }}$ May, 2003. [cited $19^{\text {th }}$ April, 2009.] http://www.dpd.cdc.gov/dpdx/html/diagnosticprocedur es.htm

Cheesebrough, M. (2004). District laboratory practice in tropical countries. Part 2. Cambridge University Press. pp. 357.

Epidi, T. T., Nwani, C. D., and Ugorji, N. P. (2008). Prevalence of malaria in blood donors in Abakaliki Metropolis, Nigeria. Scientific Research and Essay 3, 162-164.

Ghulam, M., Memon, I. A., Noorani, I.A., and Mehmood, A. K. (2004). Malaria prevalence in Sindh. Medical Channel 10, 41-42.

Guinovart, C., Navia, M. M., Tanner, M., and Alonso, P. L. (2006). Malaria: burden of disease. Current Molecular Medicine 6, 137-140. 
Ibekwe, A. C. (2004). Baseline Salmonella agglutinin titres and Plasmodium load investigation in apparently healthy freshmen in Nnamdi Azikwe University, Akwa. B.Sc. Project in the Department of Applied Microbiology and Brewing, Faculty of Natural Sciences, Nnamdi Azikwe University, Akwa. pp. 41.

Ibekwe, A. C., Okonko, I. O., Onunkwo, A. I., Ogun, A. A., Udeze, A. O., and Ejembi, J. (2009). Comparative prevalence level of Plasmodium in freshmen (first year students) of Nnamdi Azikwe University in Awka, South-Eastern, Nigeria. Malaysian Journal of Microbiology 5, 51-54.

Ibhanesebhor, S. E., Otobo, C. S., and Ladipo, O. A. (1996). Prevalence of malaria parasitaemia in transfused blood donor in Benin-City, Nigeria. Annals of Tropical Paediatrics 16, 93-95.

Kasehagen, L. J., Mueller, I., McNamara, D. T., Bockarie, M. J., Kiniboro, B., and Rare, L. (2006). Changing patterns of Plasmodium blood-stage infections in the Wosera region of Papua New Guinea monitored by light microscopy and high throughput PCR diagnosis. American Journal of Tropical of Medicine and Hygiene 75, 588-596.

Klinkenberg, E., McCall, P. J., Hastings, I. M., Wilson, M. D., Amerasinghe, F. P., and Donnelly, M. J. (2005). Malaria and irrigated crops, Accra, Ghana. Emerging Infectious Diseases 11, 1095. August 2005 [cited 18th January, 2009]. http://www.cdc.gov/ncidod/EID/vol11no08/041095.htm

Kochar, D. K., Thanvi, I., Joshi, A., Subhakaran, S. A., and Kumawat, B. L. (1998). Falciparum malaria and pregnancy. Indian Journal of Malariology 35, 123130.

Microsoft Encarta (2009) [DVD]. "Malaria." Redmond, WA: Microsoft Corporation, 2008.

Miller, L. H., Good, M. F., and Milon, G. (1994). Malaria pathogenesis. Science 264, 1878-1883.

Muhammad, A. K., Smego, R. A., Razi, S. T., and Muhammad, A. B. (2004). Emerging drug resistance and guidelines for treatment of malaria. Journal of College of Physicians and Surgeons Pakistan 14, 319-324.

Ojo, D. A., and Mafiana, C. F. (2005). Epidemiological studies of malaria parasitaemia in Abeokuta, Ogun State, Nigeria. In: the Book of Abstract of the $29^{\text {th }}$ Annual Conference \& General Meeting (Abeokuta 2005) on Microbes As Agents of Sustainable Development, organized by Nigerian Society for Microbiology (NSM), University of Agriculture, Abeokuta, 6-10 th November, 2005. pp. 50.

Okocha, C. E. C., Ibeh, C. C., Ele, P. U., and Ibeh, N. C. (2005). The prevalence of malaria parasitaeia in blood donors in a Nigerian Teaching Hospital. Journal of Vector Borne Diseases 142, 21-24.

Rahim, F., and Younas, M. (2003). Malaria in children: Clinical profile and response to treatment. A district level experience. Journal of Medical Sciences 11, 21 25.
Robert, V., Macintyre, K., Keating, J., Trape, J.F., Duchemin, J. B., and Warren, M. (2003). Malaria transmission in urban sub-Saharan Africa. American Journal of Tropical of Medicine and Hygiene 68, 169176.

Sambo, A. (2007). Why efforts to eradicate malaria fail. Pharmanews 29, 1-64.

Sabatinelli, G., Bosman, A., Lamizana, L., and Rossi, P. (1986). Prevalence of malaria in Ouagadougou and the surrounding rural environment during the period of maximal transmission. Parassitologia 28, 17-31.

SPSS (2006). Statistical Package for Social Sciences 15.0 for Windows. http://www.spss.com

Sullivan, A. D., Nyirenda, T., Cullinan, T., Taylor, T., Harlow, S. D., and James, S. A. (1999). Malaria infection during pregnancy; intrauterine growth retardation and Preterm delivery in Malawi. Journal of Infectious Diseases 179, 1580-1583.

Suh, K. N., Kain, K. C., and Keystone, J. S. (2004). Malaria. Canadian Medical Association Journal 170, 1693-1702.

Trape, J. F., and Zoulani, A. (1987). Malaria and urbanization in central Africa: the example of Brazzaville. Part III: Relationships between urbanization and the intensity of malaria transmission. Transactions of the Royal Society of Tropical Medicine and Hygiene 81, 19-25.

Vaughan, A. M., Aly, A. S. I., and Kappe, S. H. I. (2008). Malaria parasite Pre-Erythrocytic Stage Infection: Gliding and Hiding. Cell Host and Microbe 4, 209218.

World Health Organization (WHO) (1992). Expert Committee on Malaria. 19th report. WHO technical report series No. 735. Geneva.

World Health Organization (WHO) (1993). A global strategy for malaria control. World Health Organization (WHO); Geneva.

World Health Organization (WHO) (2000). Severe Falciparum Malaria. Transactions of the Royal Society of Tropical Medicine and Hygiene 94 Suppl. 1.

World Health Organization (WHO). 2005. Roll Back Malaria partnership: World Malaria Report, 2005; World Health Organization (WHO); Geneva.

World Health Organization (WHO). 2006. WHO Map of malaria endemic countries, World Health Organization (WHO); Geneva.

World Health Organization (WHO) (2007). WHO Fact sheet 94: Malaria, World Health Organization (WHO); updated May 2007.

Yusuf, M. (2007). Africa Malaria Day should focus on ridding Africa of mosquitoes. Pharma News 29, 1-64. 